\title{
A Quantum Theory Atoms in Molecules study about the inductive effect of substituents in methane derivatives
}

\section{Gabriel K. Macedo, Roberto L.A. Haiduke}

Department of Chemistry and Molecular Physics, São Carlos Institute of Chemistry, University of São Paulo, Av. Trabalhador São-Carlense, 400 - CP 780, 13560-970, São Carlos, SP, Brazil.

Equilibrium geometries and energies obtained in B2PLYP/cc-pVQZ calculations.

$\mathrm{CH}_{4}$

\begin{tabular}{|c|c|c|c|c|}
\hline Center & Atomic & Atomic & Coordinate & es (Angstror \\
\hline Number & Numbe & Type & $X$ & Z \\
\hline 1 & 6 & 0.000000 & 0.000000 & 0.000000 \\
\hline 2 & 1 & 0.626789 & 0.626789 & 0.626789 \\
\hline 3 & 1 & -0.626789 & -0.626789 & 0.626789 \\
\hline 4 & 1 & -0.626789 & 0.626789 & -0.626789 \\
\hline 5 & 1 & 0.626789 & -0.626789 & -0.626789 \\
\hline
\end{tabular}

$E=-40.4928607$ a.u

$\mathrm{CH}_{3} \mathrm{~F}$

Center Atomic Atomic Coordinates (Angstroms)

$\begin{array}{llllll}\text { Number } & \text { Number } & \text { Type } & \text { X } & \text { Y } & \text { Z }\end{array}$

$\begin{array}{rrrrrr}1 & 6 & 0 & 0.000007 & -0.634499 & 0.000000 \\ 2 & 1 & 0 & -1.028421 & -0.985762 & 0.000000 \\ 3 & 1 & 0 & 0.514156 & -0.985775 & 0.890658 \\ 4 & 1 & 0 & 0.514156 & -0.985775 & -0.890658 \\ 5 & 9 & 0 & 0.000007 & 0.751590 & 0.000000\end{array}$

$E=-139.7249346$ a.u.

$\mathrm{CH}_{2} \mathrm{~F}_{2}$

Center Atomic Atomic Coordinates (Angstroms)

$\begin{array}{llllll}\text { Number Number } & \text { Type } & \text { X } & \text { Y } & \text { Z }\end{array}$ 


$\begin{array}{llllll}1 & 6 & 0 & -0.000002 & 0.503239 & 0.000000 \\ 2 & 1 & 0 & -0.908107 & 1.100200 & 0.000000 \\ 3 & 1 & 0 & 0.908154 & 1.100116 & 0.000000 \\ 4 & 9 & 0 & -0.000002 & -0.289986 & 1.102370 \\ 5 & 9 & 0 & -0.000002 & -0.289986 & -1.102370\end{array}$

$\mathrm{E}=-238.9768327$ a.u.

$\mathrm{CHF}_{3}$

\begin{tabular}{|c|c|c|c|c|}
\hline Center & Atomic & Atomic & Coordinat & es (Angstror \\
\hline Number & Numbe & Type & $\mathrm{X}$ & Z \\
\hline 1 & 6 & 0.000000 & 0.000000 & 0.338578 \\
\hline 2 & 1 & 0.000000 & 0.000000 & 1.423988 \\
\hline 3 & 9 & 0.000000 & 1.251984 & -0.127980 \\
\hline 4 & 9 & 1.084250 & -0.625992 & -0.127980 \\
\hline 5 & 9 & -1.084250 & -0.625992 & -0.127980 \\
\hline
\end{tabular}

$\mathrm{E}=-338.2387022$ a.u.

$\mathrm{CH}_{3} \mathrm{Cl}$

\begin{tabular}{|c|c|c|c|c|}
\hline Center & Atomic & Atomic & \multicolumn{2}{|c|}{ Coordinates (Angstror } \\
\hline Number & Numbe & Type & $X$ & Z \\
\hline 1 & 6 & 0.000004 & -1.130345 & 0.000000 \\
\hline 2 & 1 & -1.027535 & -1.470975 & 0.000000 \\
\hline 3 & 1 & 0.513726 & -1.470968 & 0.889884 \\
\hline 4 & 1 & 0.513726 & -1.470968 & -0.889884 \\
\hline 5 & 17 & 0.000004 & 0.658528 & 0.000000 \\
\hline
\end{tabular}

$E=-499.99076$ a.u.

$\mathrm{CH}_{2} \mathrm{Cl}_{2}$

Center Atomic Atomic Coordinates (Angstroms) 


\begin{tabular}{cccccc} 
Number & \multicolumn{2}{l}{ Number } & Type & X & $Y$ \\
- & & & & \\
1 & 6 & 0 & 0.000000 & 0.767736 & 0.000000 \\
2 & 1 & 0 & -0.895151 & 1.372711 & 0.000000 \\
3 & 1 & 0 & 0.895132 & 1.372742 & 0.000000 \\
4 & 17 & 0 & 0.000000 & -0.216232 & 1.477304 \\
5 & 17 & 0 & 0.000000 & -0.216232 & -1.477304
\end{tabular}

$E=-959.4884244$ a.u.

\section{$\mathrm{CHCl}_{3}$}

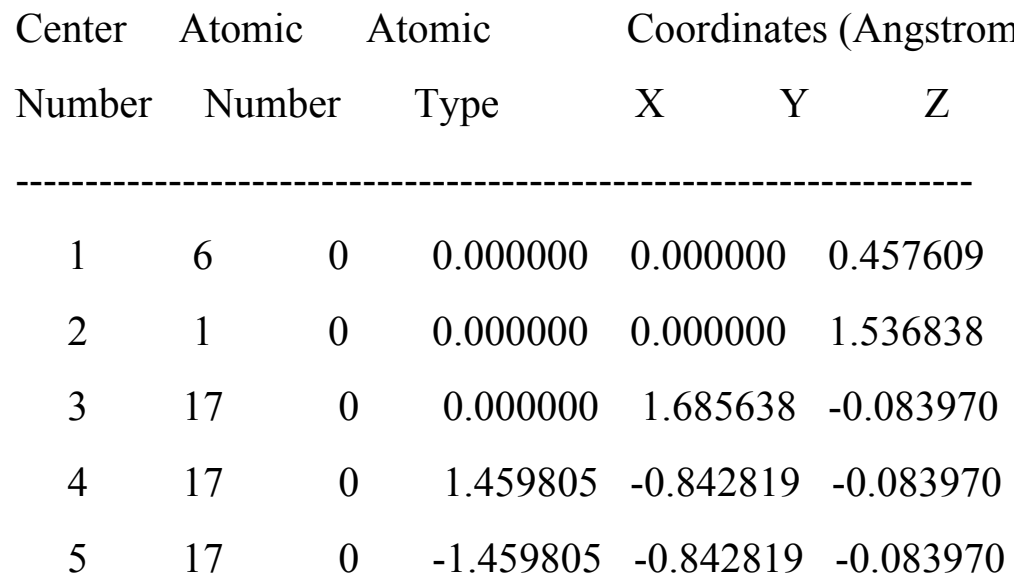

$E=-1418.9824251$ a.u.

$\mathrm{CH}_{3} \mathrm{Br}$

\begin{tabular}{cccccc} 
Center & \multicolumn{2}{c}{ Atomic } & \multicolumn{2}{c}{ Atomic } & \multicolumn{2}{c}{ Coordinates (Angstroms) } \\
Number & Number & Type & X & Y & Z \\
& & & & & \\
1 & 6 & 0 & 0.000000 & -1.524157 & 0.000000 \\
2 & 1 & 0 & -1.029667 & -1.854725 & 0.000000 \\
3 & 1 & 0 & 0.514838 & -1.854704 & 0.891722 \\
4 & 1 & 0 & 0.514838 & -1.854704 & -0.891722 \\
5 & 35 & 0 & 0.000000 & 0.420259 & 0.000000
\end{tabular}

$E=-2613.7206699$ a.u.

$\mathrm{CH}_{2} \mathrm{Br}_{2}$ 


\begin{tabular}{cccccc} 
Center & Atomic & \multicolumn{2}{c}{ Atomic } & \multicolumn{3}{c}{ Coordinates (Angstroms) } \\
Number & Number & Type & X & Y & $Z$ \\
--------------------------------------------------------------- \\
1 & 6 & 0 & 0.000000 & 0.932759 & 0.000000 \\
2 & 1 & 0 & -0.896113 & 1.533961 & 0.000000 \\
3 & 1 & 0 & 0.896110 & 1.533964 & 0.000000 \\
4 & 35 & 0 & 0.000000 & -0.123778 & 1.617847 \\
5 & 35 & 0 & 0.000000 & -0.123778 & -1.617847
\end{tabular}

$\mathrm{E}=-5186.9459748$ a.u.

$\mathrm{CHBr}_{3}$

Center Atomic Atomic Coordinates (Angstroms)

Number Number Type $\quad$ X $\quad$ Y $\quad$ Z

$\begin{array}{cccccc}1 & 6 & 0 & 0.000000 & 0.000000 & 0.522438 \\ 2 & 1 & 0 & 0.000000 & 0.000000 & 1.600445 \\ 3 & 35 & 0 & 0.000000 & 1.847283 & -0.045096 \\ 4 & 35 & 0 & 1.599794 & -0.923642 & -0.045096 \\ 5 & 35 & 0 & -1.599794 & -0.923642 & -0.045096\end{array}$

$E=-7760.1669453$ a.u.

$\mathrm{CH}_{2} \mathrm{O}$

\begin{tabular}{cccccc} 
Center & \multicolumn{2}{c}{ Atomic } & \multicolumn{2}{c}{ Atomic } & \multicolumn{3}{c}{ Coordinates (Angstroms) } \\
Number & Number & Type & X & Y & Z \\
-------------------------------------------------------------- \\
1 & 6 & 0 & 0.000000 & 0.000000 & -0.528418 \\
2 & 1 & 0 & 0.000000 & 0.935164 & -1.111388 \\
3 & 1 & 0 & 0.000000 & -0.935164 & -1.111388 \\
4 & 8 & 0 & 0.000000 & 0.000000 & 0.674161
\end{tabular}

$\mathrm{E}=-114.481309$ a.u.

$\mathrm{CH}_{2} \mathrm{~S}$ 


\begin{tabular}{cccccc} 
Center & \multicolumn{2}{c}{ Atomic } & \multicolumn{2}{c}{ Atomic } & \multicolumn{2}{c}{ Coordinates (Angstroms) } \\
Number & Number & Type & X & Y & Z \\
--------------------------------------------------------------- \\
1 & 6 & 0 & 0.000000 & 0.000000 & -1.025446 \\
2 & 1 & 0 & 0.000000 & 0.920287 & -1.600559 \\
3 & 1 & 0 & 0.000000 & -0.920287 & -1.600559 \\
4 & 16 & 0 & 0.000000 & 0.000000 & 0.584612
\end{tabular}

$E=-437.3456985$ a.u.

$\mathrm{CH}_{3} \mathrm{Li}$

\begin{tabular}{crrrrr} 
Center & Atomic & \multicolumn{2}{c}{ Atomic } & \multicolumn{2}{c}{ Coordinates (Angstroms) } \\
Number & Number & Type & X & Y & Z \\
- & & & & & \\
1 & 6 & 0 & 0.000000 & 0.000000 & 0.387671 \\
2 & 1 & 0 & 0.000000 & 1.011334 & 0.807139 \\
3 & 1 & 0 & 0.875841 & -0.505667 & 0.807139 \\
4 & 1 & 0 & -0.875841 & -0.505667 & 0.807139 \\
5 & 3 & 0 & 0.000000 & 0.000000 & -1.582481
\end{tabular}

$E=-47.3612794$ a.u.

$\mathrm{CH}_{3} \mathrm{Na}$

\begin{tabular}{cccccc} 
Center & \multicolumn{2}{c}{ Atomic } & \multicolumn{2}{c}{ Atomic } & \multicolumn{2}{c}{ Coordinates (Angstroms) } \\
Number & Number & Type & X & Y & Z \\
& & & & & \\
\hline 1 & 6 & 0 & 0.000000 & 0.000000 & -1.221412 \\
2 & 1 & 0 & 0.000000 & 1.024226 & -1.595831 \\
3 & 1 & 0 & -0.887006 & -0.512113 & -1.595831 \\
4 & 1 & 0 & 0.887006 & -0.512113 & -1.595831 \\
5 & 11 & 0 & 0.000000 & 0.000000 & 1.101452
\end{tabular}

$E=-202.0321151$ a.u.

\section{$\mathrm{CH}_{2} \mathrm{ClF}$}




\begin{tabular}{cccccc} 
Center & \multicolumn{2}{c}{ Atomic } & \multicolumn{2}{c}{ Atomic } & \multicolumn{2}{c}{ Coordinates (Angstrom } \\
Number & Number & Type & X & Y & Z \\
& & & & & \\
\hline 1 & 6 & 0 & 0.566075 & 0.573724 & -0.000001 \\
2 & 1 & 0 & 0.675170 & 1.164063 & -0.901788 \\
3 & 1 & 0 & 0.675147 & 1.164005 & 0.901827 \\
4 & 17 & 0 & -1.072099 & -0.117238 & -0.000001 \\
5 & 9 & 0 & 1.497658 & -0.419706 & -0.000002
\end{tabular}

$E=-599.2298964$ a.u.

$\mathrm{CHClF}_{2}$

\begin{tabular}{cccccc} 
Center & Atomic & \multicolumn{2}{c}{ Atomic } & \multicolumn{2}{c}{ Coordinates (Angstroms) } \\
Number & Number & Type & $\mathrm{X}$ & $\mathrm{Y}$ & $\mathrm{Z}$ \\
& & & & & \\
1 & 6 & 0 & 0.441771 & 0.000000 & 0.365104 \\
2 & 1 & 0 & 0.547370 & -0.000001 & 1.443875 \\
3 & 17 & 0 & -1.279863 & 0.000000 & -0.050639 \\
4 & 9 & 0 & 1.031093 & 1.085568 & -0.154091 \\
5 & 9 & 0 & 1.031094 & -1.085567 & -0.154091
\end{tabular}

$E=-698.4817228$ a.u.

$\mathrm{CHFCl}_{2}$

\begin{tabular}{|c|c|c|c|c|}
\hline Center & Atomic & Atomic & Coordinates & s (Angstro1 \\
\hline Number & Numbe & Type & $X$ & Z \\
\hline 1 & 6 & -0.178074 & 0.521500 & 0.000000 \\
\hline 2 & 1 & -1.072052 & 1.130527 & 0.000000 \\
\hline 3 & 9 & 0.910555 & 1.314616 & 0.000000 \\
\hline 4 & 17 & -0.178074 & -0.473267 & 1.462838 \\
\hline 5 & 17 & -0.178074 & -0.473267 & -1.462838 \\
\hline
\end{tabular}

$E=-1058.729528$ a.u.

$\mathrm{CH}_{3} \mathrm{CH}_{3}$ 


\begin{tabular}{|c|c|c|c|c|}
\hline Center & Atomic & Atomic & Coordinate & es (Angstron \\
\hline Number & Numbe & Type & $X$ & $\mathrm{Z}$ \\
\hline 1 & 6 & 0.000000 & 0.000000 & -0.762277 \\
\hline 2 & 1 & -0.507072 & 0.878274 & -1.157569 \\
\hline 3 & 1 & -0.507072 & -0.878274 & -1.157569 \\
\hline 4 & 1 & 1.014144 & 0.000000 & -1.157569 \\
\hline 5 & 6 & 0.000000 & 0.000000 & 0.762290 \\
\hline 6 & 1 & -1.014149 & 0.000000 & 1.157543 \\
\hline 7 & 1 & 0.507074 & -0.878279 & 1.157543 \\
\hline 8 & 1 & 0.507075 & 0.878279 & 1.157543 \\
\hline
\end{tabular}

$\mathrm{E}=-79.7839507$ a.u.

$\mathrm{CH}_{3} \mathrm{OCH}_{3}$

\begin{tabular}{cccccc} 
Center & Atomic & \multicolumn{2}{c}{ Atomic } & \multicolumn{3}{c}{ Coordinates (Angstroms) } \\
Number & Number & Type & $\mathrm{X}$ & $\mathrm{Y}$ & $\mathrm{Z}$ \\
\hline 1 & 8 & 0 & 0.000000 & 0.000000 & 0.591208 \\
2 & 6 & 0 & 0.000000 & 1.167802 & -0.196081 \\
3 & 6 & 0 & 0.000000 & -1.167802 & -0.196081 \\
4 & 1 & 0 & 0.000000 & 2.017127 & 0.480010 \\
5 & 1 & 0 & 0.000000 & -2.017127 & 0.480010 \\
6 & 1 & 0 & 0.887840 & 1.218901 & -0.834178 \\
7 & 1 & 0 & -0.887840 & 1.218901 & -0.834178 \\
8 & 1 & 0 & -0.887840 & -1.218901 & -0.834178 \\
9 & 1 & 0 & 0.887840 & -1.218901 & -0.834178
\end{tabular}

$\mathrm{E}=-154.9775889$ a.u.

$\mathrm{CH}_{3} \mathrm{OH}$

Center Atomic Atomic Coordinates (Angstroms)

Number Number Type $\quad$ X $\quad$ Y $\quad$ Z 


$\begin{array}{llllll}1 & 6 & 0 & 0.046380 & 0.663440 & 0.000000 \\ 2 & 1 & 0 & -0.436575 & 1.074872 & 0.888021 \\ 3 & 1 & 0 & -0.436575 & 1.074872 & -0.888021 \\ 4 & 1 & 0 & 1.085649 & 0.976794 & 0.000000 \\ 5 & 8 & 0 & 0.046380 & -0.755839 & 0.000000 \\ 6 & 1 & 0 & -0.861823 & -1.060464 & 0.000000\end{array}$

$E=-115.696632$ a.u.

$\mathrm{CH}_{3} \mathrm{SH}$

\begin{tabular}{cccccc} 
Center & Atomic & \multicolumn{2}{c}{ Atomic } & \multicolumn{3}{c}{ Coordinates (Angstrom } \\
Number & Number & Type & $\mathrm{X}$ & $\mathrm{Y}$ & $\mathrm{Z}$ \\
& & & & & \\
1 & 6 & 0 & 0.047763 & 1.154166 & 0.000000 \\
2 & 1 & 0 & 1.090512 & 1.456039 & 0.000000 \\
3 & 1 & 0 & -0.430640 & 1.547197 & 0.890524 \\
4 & 1 & 0 & -0.430640 & 1.547197 & -0.890524 \\
5 & 16 & 0 & 0.047763 & -0.665250 & 0.000000 \\
6 & 1 & 0 & -1.280016 & -0.831436 & 0.000000
\end{tabular}

$E=-438.5789659$ a.u.

$\mathrm{CH}_{3} \mathrm{NH}_{2}$

\begin{tabular}{crrrrr} 
Center & \multicolumn{2}{c}{ Atomic } & \multicolumn{2}{c}{ Atomic } & \multicolumn{3}{c}{ Coordinates (Angstrom } \\
Number & Number & Type & X & $Y$ & $Z$ \\
& & & & & \\
1 & 6 & 0 & -0.049839 & 0.704689 & 0.000000 \\
2 & 1 & 0 & -0.587654 & 1.062011 & 0.875195 \\
3 & 1 & 0 & -0.587654 & 1.062011 & -0.875195 \\
4 & 1 & 0 & 0.941066 & 1.168749 & 0.000000 \\
5 & 7 & 0 & -0.049839 & -0.757233 & 0.000000 \\
6 & 1 & 0 & 0.441076 & -1.110135 & -0.809148 \\
7 & 1 & 0 & 0.441076 & -1.110135 & 0.809148
\end{tabular}

$E=-95.8227892$ a.u. 
$\mathrm{CH}_{3} \mathrm{NO}_{2}$

\begin{tabular}{|c|c|c|c|c|}
\hline Center & Atomic & Atomic & Coordinate & es (Angstron \\
\hline Number & Number & Type & $X$ & Z \\
\hline 1 & 6 & 0.038416 & -1.319909 & 0.000000 \\
\hline 2 & 1 & 1.072469 & -1.633982 & 0.000000 \\
\hline 3 & 1 & -0.488958 & -1.653709 & 0.885967 \\
\hline 4 & 1 & -0.488958 & -1.653709 & -0.885967 \\
\hline 5 & 7 & 0.000000 & 0.171265 & 0.000000 \\
\hline 6 & 8 & -1.107675 & 0.689157 & 0.000000 \\
\hline 7 & 8 & 1.067044 & 0.768592 & 0.000000 \\
\hline
\end{tabular}

$\mathrm{E}=-244.9764289$ a.u. 